\title{
Rapid diagnosis of bacteric meningitis
}

\author{
Sara Giordana Rimoldi, Cristina Pagani, Loredana Tocalli, Alessandra Lombardi, Maria Rita Gismondo \\ Unità Operativa di Microbiologia e Virologia A.O. e Polo Universitario L. Sacco, Milano
}

Key words: Meningite batterica, Real time detection, Microscopico diretto, Ricerca antigenica

Uso della real time nella diagnosi della meningite

\section{SUMMARY}

Gram stain and bacterial antigen detection are economic and rapid tests for the identification of the bacteria in cerebrospinal fluid (CSF) for the diagnosis of meningitis. Even if culture is the gold standard of these diagnosis, it requires long time (growth time).

The purpose of our study was to compare in the laboratory diagnosis of meningitis real time PCR (Eurospital, Italy). with standard methods. Samples were collected in patients referred to L. Sacco Universital Hospital (range of age: 16 to 70 years) from September 2008 to July 2009. 9 out of I 30 CFS samples investigated were positive in PCR but they produced different results to the other methods used.

\section{INTRODUZIONE}

La meningite batterica è un'emergenza microbiologica causata da diversi patogeni Gram positivi e Gram negativi (1). Diverse sono le tecnologie diagnostiche, tradizionali ed innovative, utilizzate negli ultimi anni per la diagnosi rapida di meningite (2). Tutt'oggi il gold standard rimane l'esame colturale anche se tale test necessita tempi lunghi di crescita e spesso, a fronte di una terapia antibiotica pregressa, dia difficoltà di crescita batterica. Al fine di valutare sensibilità e specificità dei diversi metodi indagati, abbiamo voluto comparare i dati ottenuti con il metodo real time PCR (RT-PCR) a quelli dell'esame microscopico diretto e della ricerca antigenica (3).

\section{METODI}

Tra Settembre 2008 e Luglio 2009 all’U.O. di Microbiologia e Virologia dell'A.O. e Polo Universitario L. Sacco sono pervenuti 130 campioni di liquor di pazienti provenienti dal PS e dai reparti di area critica ( 80 maschi, 50 femmine) in età compresa tra 16-70 anni. La diagnosi è stata eseguita mediante esame microscopico diretto eseguito con colorazione di Gram, colturale (Dasit, Milano-Italia) e ricerca degli antigeni solubili Wellcogen (DID, Milano-Italia) specifici per Neisseria meningitidis, Streptococcus pneumoniae, Haemophilus influenzae, Streptococcus agalactiae e Escherichia coli.

I liquor sono stati, inoltre, testati parallelamente con RT-PCR (Eurospital) in grado di identificare in sole 3 ore, direttamente da campione biologico, la presenza di $N$. meningitidis, $H$. influenzae, S. pneumoniae, Listeria spp, E. coli, S. agalactiae ed Adenovirus.

\section{RISULTATI}

Dei 130 liquor indagati, 9 sono stati i casi positivi confermati alla RT-PCR: ma con sensibilità diverse ai metodi indagati (tabella 1).
Di 3 campioni positivi per $N$. meningitidis all'esame microscopico diretto e al RT-PCR solo 1 ha trovato conferma anche agli altri due i metodi: 2 sono risultati negativi all'esame colturale, 1 positivo agli antigeni solubili.

Analogamente 3 campioni positivi all'esame microscopico e colturale per $S$. pneumoniae non hanno mostrato positività agli antigeni solubili; 2 campioni positivi all'esame colturale, rispettivamente per Listeria monocytogenes e $H$. influenzae, non hanno trovato conferma all'esame microscopico diretto e agli antigeni solubili. In un caso, di paziente adulto, positivo alla RT-PCR per E. coli è risultato negativo all'esame colturale e agli antigeni solubili.

Dieci campioni negativi all'esame microscopico e colturale sono stati testati come pool di controllo in biologia molecolare.

\section{CONCLUSIONI}

Nella nostra osservazione preliminare la RT-PCR si è mostrata un ottimo strumento di diagnosi rapida della meningite, soprattutto nelle situazioni cliniche in cui la carica batterica non è elevata e una pregressa terapia antibiotica potrebbe impedire la crescita batterica in coltura.

\section{BIBLIOGRAFIA}

1. Dobson R, Shannon C. A 56 yrs old man with headache, fever and neurological symtoms.

2. BMJ. 2010 Jan 20; 340: b5603. doi: 10.1136/bmj.b5603. No abstract available.

3. Levy C, et al. Surveillance network of bacterial meningitis in children, 7 years of surveillance in France. Arch Pediatr 2008 Dec; 15 Suppl 3: S 99S104.

4. Roos KL, Van de Beek D. Bacterial meningitis. Handb Clin Neurol. 2010; 96C: 51-63. Epub 2010 Jan 19.

Tabella I. Positività riscontrata nei diversi metodi utilizzati per la diagnosi di meningite.

\begin{tabular}{lllll}
\hline Patogeno & Esame microscopico & Esame colturale & Antigeni & RT-PCR \\
\hline N. meningitidis & Diplococchi Gram negativi & negativo & Positivo per N. meningitidis & Positivo per N. meningitidis \\
\hline N. meningitidis & Diplococchi Gram negativi & negativo & negativo & Positivo per N. meningitidis \\
\hline N. meningitidis & Diplococchi Gram negativi & Positivo per N. meningitidis & negativo & Positivo per N. meningitidis \\
\hline S. pneumoniae & Diplococchi Gram positivi & Positivo per S. pneumoniae & negativo & Positivo per S. pneumoniae \\
\hline S. pneumoniae & Diplococchi Gram positivi & Positivo per S. pneumoniae & negativo & Positivo per S. pneumoniae \\
\hline S. pneumoniae & Diplococchi Gram positivi & Positivo per S. pneumoniae & negativo & Positivo per S. pneumoniae \\
\hline L. monocytogenes & negativo & Positivo per L. monocytogenes & negativo & Positivo per L. monocytogenes \\
\hline H. influenzae & negativo & Positivo per H. influenzae & negativo & Positivo per H. influenzae \\
\hline E. coli & negativo & Positivo per E. coli & Positivo per E. coli & Positivo per E. coli
\end{tabular}

\section{Corresponding author: Sara Giordana Rimoldi}

Tel.: +3902 39042479

E-mail: Rimoldi.sara@hsacco.it 